\title{
Private Pensions in Albania (The Need of a Reform According to the European Standards)
}

\author{
LI.M. Ina Petraj \\ Law Faculty, Vlora University Albania \\ ina.petraj@unitir.edu.al
}

Doi:10.5901/ajis.2013.v2n2p237

\begin{abstract}
During the midlife age nobody is concerned or worried about the pensions. Years pass and pensions become our main common concern. We start questioning ourselves if we will have a sufficient pension that will allow us a good quality of life and if the amount of our monthly contributions will gave us a right pension. The background of this research was inspired from the questions that were mentioned previously and also from the fact that the work of our whole life needs to be awarded with a good pension. The supplementary income that we can have in addition to the income that derives from the payment of monthly contributions is really tempting. The investment in voluntary pensions can be a complementary asset to the actual system "pay as you go". The purpose and the functioning of the voluntary pensions are sometimes perceived as ambiguous. The supervision institutions and the law "Voluntary Pension Funds" help the third age. We can have a major return from investing our money in the long-term; we also have a supplementary income at the moment of our retirement having the benefits of good life at an old age. The number of the contributors that have invested a part of their savings in voluntary pension funds has increased according to the recent statistical data. The quantity of the amount of individual contribution has increased too. Albanians are conscious that a part of their savings should be a long-term risk free investment.
\end{abstract}

Keywords: Supervision Authority, Pension Fund, Administration Society, European Union Directives.

\section{Introduzione}

Questo elaborato, è frutto della ricerca verso lo studio di un ramo del mercato finanziario non bancario in Albania: II mercato delle pensioni private supplementari. L'analisi e lo studio nel campo delle pensioni supplementari è indispensabile per la riforma del sistema pensionistico in Albania in armonia con le direttive dell'Unione Europea per permettere lo sviluppo di questo settore ibrido e nuovo nella legislazione Albanese.

Durante il mio studio mi sono soffermata particolarmente nella descrizione dettagliata della legislazione Albanese, nel sistema della vigilanza dei fondi pensione, nonchè nel loro rapporto con l'acquis communautaire. Noi siamo un paese giovane con poca esperienza e storia nella regolamentazione di questo mercato, perciò il sostegno dei partner internazionali ha contibuito indispensabilmente nella istituzione del mercato dei fondi pensione. Oltre le direttive dell'Unione Europea, le norme sulle pensioni private devono adattarsi anche ai principi internazionali della vigilanza dei fondi pensione, sanzionati dall'International Organisation of Pension Supervisors (IOPS), nonchè ai principi OECD'1.

Attraverso la descrizione, la comparazione e l'analisi, cercherò di dare una risposta alle problematiche legali più importanti del sistema.

\section{La storia dello "schema delle pensioni"}

La definizione dei tre schemi o pilastri delle pensioni, ha un ruolo integrante per questa ricerca, facendo riferimento alla terminologia sulla quale si è basata anche la stessa Banca Mondiale essi vengono definiti come segue.

Lo schema delle pensioni, si considerano i risparmi collettivi attraverso i quali i contribuenti percepiscono un reddito alla loro andata in pensione.

${ }^{1}$ The Organisation for Economic Co-operation and Development 
La misura del contributo, si intende la soglia del contributo attuale, e si definisce come una percentuale della retribuzione del lavoratore. In uno determinato sistema si potrebbe applicare la stessa soglia di contributo per tutti i contribuenti. Lo schema PAYG richiede che il contributo viene versato da entrambe le parti, sia dal lavoratore che dal datore di lavoro, con lo scopo di contribuire allo sistema attuale della previdenza sociale.

In conclusione, il contributo si considera come prelievo obbligatoria sulla retribuzione a favore dello Stato, con una deteminata incidenza percentuale, il quale viene pagato dalle persone che producono un reddito. I contributi si pagano in base alla legge n. 7703/1993 "In materia della previdenza sociale nella Repubblica Albanese".

Le assicurazioni sociali vengono pagate dai contributi dei lavoratori assicurati. I lavoratori e i datori di lavoro contribuiscono attraverso i contributi mensili di assicurazioni sociali per le pensioni. In altre parole, questo sistema viene conosciuto come "pay as you go". Questo sistema assegna le indennità per l'inabilità temporanea a causa delle malattie, maternità, morte, le pensioni di anzianità, gli infortuni sul lavoro, malattie professionali e disoccupazione. Uno dei punti forti dello schema PAYG è rappresentato dal metodo di finanziamento, attraverso il quale le spese per la riscossione delle pensioni attuali vengono sottratte alle entrate attuali, spesso finanziate dalle imposte applicate ai redditi. Occorre sottolineare che lo schema PAYG è totalmente non finanziata, ma gestita in modo pubblico. In altre parole lo schema assume la responsabilità di garantire la pensione ai contribuenti una volta raggiunta l'età, ma non accantona i fondi che possono essere utilizzati per spese od oneri che possono emergere nel futuro. PAYG si basa sulla "solidarietà tra le generazioni" dove i lavoratori di oggi sostengono i lavoratori che hanno raggiunto l'età pensionabile.

Sulla scia degli schemi delle pensioni, accanto alla PAYG esiste anche lo "schema finanziato" (funded scheme) conosciuto come il secondo pilastro, il quale ti permette di optare per una distribuzione della spesa del denaro per tutta la vita. II secondo pilastro e una combinazione tra lo schema privato e pubblico delle pensioni basato sulla capitalizzazione e la redistribuzione, ma che deve essere nettamente separato dal primo pilastro, per di più deve essere dal punto di vista finanziario molto stabile. Secondo questo schema, una parte del contributo viene gestito dallo schema pubblico "pay as you go" (lo schema attuale basato sulla solidarietà tra le generazioni), la quale garantisce una pensione base, mentre l'altra parte viene gestita dalle companie private, che saranno scelte dallo stato, in base alla legislazione che entrerà in vigore. II denaro che e stato pagato in forma dei contributi obbligatori ed il profitto realizzato dalle companie privati, quest'ultime lo erogeranno al raggiungimento dell'età pensionabile.

II terzo pilastro viene conosciuto come "lo schema dei contributi definiti" (defined contribution) la quale e interamente finanziata. Questo e uno schema gestito dai privati, ma preliminarmente approvata dall'Autorità di Vigilanza Finanziaria (AVF) sotto la continua vigilanza da parte di quest'ultimo.

Secondo le valutazioni effettuate dalla Banca Mondiale lo schema dei contributi definiti, viene chiamata anche lo schema di accumulazione di denaro. II beneficio che viene erogato nella forma delle pensioni si basa sul valore della contribuzione attuale più gli interessi maturati, che derivano dall'investimento dei contributi da parte delle società di gestione.

A differenza dello schema PAYG, i benefici non sono totalmente determinati, ma dipendono dal livello dei contributi e dal tasso di ritorno dell'investimento. Questi schemi garantiscono ai contribuenti un importo che può essere utilizzato per il soddisfacimento delle esigenze finanziarie al mometo dell'età pensionabile.

\section{II Sistema pensionistico in Albania}

Il sistema delle pensioni in Albania si compone dallo schema pubblico pay as you go (il primo pilastro) gestito dall'Istituto Nazionale della Previdenza Sociale (INPS in Albanese ISSH) nonche dallo schema privato (terzo pilastro) gestito dalle società di amministrazione dei fondi pensione.

Lo schema pubblico "pay as you go" si basa sul modello di Bismarckiano, dove i contributi e i benefici si determinano in rapporto alla retribuzione e al principio di solidarietà.

La riforma delle pensioni in Albania si e caratterizzata da due interventi legislativi:

- La riforma strutturale del 1993

- La riforma parametrica del 2002.

Le trasformazioni politiche e socio-economiche degli anni 90 hanno imposto la necessità di una riforma radicale nel sistema della previdenza sociale. Sostanzialmente attraverso la riforma si cercava di costituire un sistema stabile ed efficiente, in grado di affrontare i problemi emergenti del tempo con l'obiettivo di servire anche alle generazioni future. Queste sono state le ragioni che hanno indotto il legislatore ad approvare nel 11 maggio 1993 la legge n. 7703 "in materia previdenziale in Albania" entrato in vigore nel mese di ottobre dello stesso anno. La riforma del 1993 si 
considera "strutturale", in quanto ha riorganizzato il sistema della previdenza sociale adattandolo all'economia di mercato. Questa riforma ha istituito la struttura attuale dello schema pay as you go, in Albania. La seconda riforma del sistema pensionistico 2002 fu del tipo parametrico. Essa fu sostenuta dalla Banca Mondiale con le finalità di crescere la stabilità del sistema.

Lo schema privato delle pensioni, anche se de jure fu presentata nel 1995, iniziò a funzionare soltanto dopo dieci anni. Nel 2005 furono autorizzate le prime società che hanno gestito i fondi pensione privati. Lo schema privato delle pensioni (terzo pilastro) funziona come uno schema supplementare di quello pubblico. Essa e completamente facoltativa, e si rivolge alle persone che desiderano avere una pensione aggiuntiva, rispetto alla pensione erogata dagli enti pubblici.

Le pensioni pubbliche in Albania, riferendosi agli indici principali del sistema, continuano a rimanere a livelli preoccupanti. Oggi si contano più di 560 milla pensionati coinvolti nello schema, i quali rappresentano il $21 \%$ della popolazione residenziale in Albania. Questo è un indice che tende di aumentare nei prossimi anni. Lo stato Albanese spende circa 95 miliardi di lekë ${ }^{2}$ per il sistema delle pensioni, una cifra molto elevata, tenendo conto che essa rappresenta $85 \%$ della spesa totale nel sistema previdenziale. I dati riportati ci dimostrano le problematiche serie del sistema previdenziale nazionale, con l'impossibilità di assicurare l'autosufficienza tramite lo schema pubblico. I problemi riscontrati nel sistema previdenziale, sono causati in primo luogo dal basso livello delle entrate (contributi) in rapporto con il Prodotto Interno Lordo (PIL) il quale ha inciso negativamente. In secondo luogo, il rapporto molto critico tra i contribuenti e beneficiari. Attualmente questo rapporto si attesta 1.2:1, il che vuol dire, che da 1,2 contribuenti del sistema ne beneficiano soltanto 1. Mentre il rapporto ottimale per il funzionamento del sistema è 4:1. Infine, il basso rapporto tra pensione/retribuzione che è di 30\%-35\% piuttosto di 70\%-75\% (tasso legale).

A questo punto, ci sono tre le problematiche da risolvere. La demotivazione della nuova generazione per far parte al sistema previdenziale pubblico, in quanto offre una pensione bassa e richiede un alto tasso di contributi. II malfunzionamento per più di 15 anni del sistema previdenziale complementare, i problemi di disoccupazione, informalità e specialmente l'emigrazione hanno diminuito il tasso dei contribuenti negli ultimi 20 anni, il quale crea preoccupazione non solo per il funzionamento del sistema, ma anche per il suo impatto sociale nel futuro. Sfortunatamente, il sistema pensionistico richiede un tasso di contributi relativamente alto ed offre un importo delle pensioni basso.

Partendo da questi dati, vorrei soffermarmi alla seconda problematica, il malfunzionamento del sistema previdenziale complementare ed il suo impatto nel sistema pensionistico generale in Albania.

Occorre precisare che negli ultimi anni dello scorso secolo, molti paesi europei hanno avuto seri problemi con il sistema previdenziale pubblico. La maggioranza dei paesi ha risolto le problematiche attraverso l'aumento dell'età pensionabile o dell'importo dei contributi. In entrambi i casi, l'applicazione delle riforme ha avuto delle grosse difficoltà. Inoltre, le riforme intraprese non hanno risolto i problemi, ansi hanno aggravato sempre di più il rapporto tra le generazioni, aumentando l'importo dei contributi nei confronti delle giovani generazioni. La Banca Mondiale ed altre organizzazioni internazionali in queste condizioni hanno proposto una nuova risoluzione del problema quale la combinazione tra il sistema previdenziale pubblico con quello privato (obbligatorio e complementare), responsabilizzando gli individui nella risoluzione dei problemi sociali. In molti paesi, accanto al sistema previdenziale pubblico, si istituirono anche sistemi previdenziali delle pensioni supplementari privati e dopo il 1998 anche sistemi previdenziali obbligatori privati. Oggi questi sistemi esistono in tutto il mondo ed hanno avuto successo.

La riforma nel sistema pensionistico ha coinvolto anche l'Albania nel 2002. Essa ha determinato un aumento dell'età pensionabile a 60 anni per le donne e 65 anni per gli uomini, adattandosi all'età pensionabile dei paesi dell'Unione Europea, come un requisito indispensabile per la nostra adesione all'UE. Questa riforma è ancora in corso e non ha esaurito tutte le categorie. A mio avviso, l'aumento dell'età pensionabile non è l'unica soluzione per il sistema pensionistico pubblico, poiché, secondo INSTAT l'età media nel nostro paese è 31 anni rispetto a 48 anni di quella europea ed una riforma del genere non determinerebbe nessun migloramento del sistema. II nostro legislatore deve fare di più riguardo i fenomeni sociali ed economici come: disoccupazione, informalità, emigrazione ecc. per avere risultati concreti nel sistema piuttosto di aumentare l'età pensionabile. Inoltre, l'aumento dell'età pensionabile potrebbe causare un impatto negativo all'economia del paese aumentando il tasso di disoccupazione giovanile.

${ }^{2}$ La moneta nazionale Albanese. 


\section{Le pensioni complementari}

A causa dei cambiamenti demografici in corso, dove la popolazione di terza età è in progressiva crescita, è impossibile per il sistema pensionistico pubblico che si basa sulla solidarietà tra le generazioni di garantire a questa categoria una serena vita finanziaria. Per queste ragioni, oltre al sistema pensionistico pubblico, sarebbe necessario l'istituzione del sistema previdenziale complementare, inizialmente a carattere integrativo, e successivamente perché no, a carattere prevalente. Secondo la normativa, questo sistema svolge due funzioni; in primo luogo eroga le pensioni integrative oltre la pensione di base del sistema previdenziale obbligatorio, in secondo luogo, le pensioni vengono erogate a condizioni più favorevoli rispetto al sistema obbligatorio. Pertanto, il legislatore deve sostenere al massimo questo modello, applicando un sistema fiscale più favorevole ai contribuenti esonerandoli dall'imposta sul reddito, così come funziona nella gran parte dei paesi del mondo. Sulla stessa direzione la legge Albanese sancisce che i contributi versati sui fondi pensione vengono detrati dall'imposta sul reddito (art. 88).

Basandosi sull'esperienza dello sviluppo dei sistemi pensionistici, sono due i modelli principali istituiti in base alle condizioni sociali di ogni paese. II primo modello che è anche il più diffuso si compone da tre pilastri. II primo pilastro è il sistema previdenziale obbligatorio (PAYG) il quale copre il 40\%-45\% delle pensioni. II secondo pilastro rappresentato dal settore privato obbligatorio, il quale copre il $20 \%-25 \%$ delle pensioni ed il terzo pilastro denominato come il sistema pensionistico complementare il quale copre il 10\%-15\% delle pensioni. In questo modo, ogni persona che andrà a pensione può beneficiare da entrambi i sistemi. II secondo modello pensionistico si compone invece di due pilastri. Nel primo pilastro ne fa parte il sistema previdenziale obbligatorio, il quale copre il $65 \%$ delle pensioni ed il secondo pilastro dal sistema previdenziale complementare che copre il $20 \%$ delle pensioni. Entrambi i modelli in vari paesi sono risultati di successo rispetto al vecchio sistema pensionistico pubblico. Questo per vari ragioni, tra le quali, il sistema privato risolve il problema demografico, stimola il mercato di lavoro, nonché esclude il Governo nella gestione del sistema stesso.

In Albania, attualmente opera il secondo modello, ma subito dopo l'implementazione della riforma del sistema pensionistico da parte del Parlamento Albanese, il sistema funzionerà secondo il primo modello. Il sistema delle pensioni sarà composto da tre pilastri, il quale per il nostro paese sembra più adeguato, tenendo conto della popolazione giovane e del basso tasso di sostituibilità (retribuzione/pensione).

La riforma ha come finalità la creazione di un sistema pensionistico autosufficiente, minimizzando così i costi di sostegno che gravano sul bilancio dello stato, il quale finanzia una buona parte del sistema. L'altra finalità è lo stimolo dei lavoratori di finanziare in questo sistema, con l'obiettivo l'aumento dei benefici al tempo quando andranno a pensione in proporzione con i contributi versati durante gli anni lavorativi.

Così come abbiamo già menzionato, il nuovo sistema pensionistico si basa sui i tre pilastri e rende più effettivo $\mathrm{e}$ incentivante il sistema stesso. Non è previsto un nuovo cambiamento dell'età pensionabile, in quanto essa risulta in armonia con quella dei paesi dell'Unione Europea. II primo pilastro, il cosiddetto "colonna zero" sono inclusi tutti gli anziani indipendentemente dal fatto se contribuiscono 0 meno, proteggendoli così dalla povertà. Questo pilastro è obbligatorio e viene gestito completamente dallo Stato. Anche se i benefici sono relativamente bassi, si considera un pilastro sicuro. II secondo pilastro è una combinazione tra il sistema pensionistico pubblico e privato. Le companie private saranno scelte dallo Stato nel pieno rispetto delle regole imposte dalla legislazione vigente, determinando così un aumento del reddito delle persone in quel periodo della vita quando non saranno più in grado di lavorare. II terzo pilastro è completamente privato e si basa solamente nella volontà delle persone di avere o meno una pensione maggiore rispetto a quella ordinaria.

In Albania le pensioni private volontarie, sono regolate dalla legge n. 10197/2009 "In materia dei fondi pensione volontari". Lo scopo principale di questa legge è l'emanazione delle regole e di standard, la rigorosa applicazione delle quali garantirà un sistema finanziario stabile ed efficiente. Vengono regolati innazittutto, la costituzione, l'attività e la vigilanza dei fondi pensione a contributi determinati. ${ }^{3}$ La nuova legge è suddivisa in 17 sezioni e regola dei concetti riguardanti i fondi pensione, le società di amministrazione, depositario, agente, fondi pensione per il liberi professionisti, gli investimenti dei fondi di investimento, il loro marketing, le tariffe delle società di amministrazione, vigilanza, tassazione, reati finanziari e riciclaggio del denaro ecc. 
L'Autorità di Vigilanza Finanziaria (AVF) è l'unica autorità competente per l'autorizzazione, vigilanza e regolamentazione dei fondi pensione privati. Dall'AVF vengono autorizzate le società di amministrazione le quali svolgono le seguenti attività: la raccolta, la gestione e l'investimento del patrimonio dei fondi pensione privati, la realizzazione dei profitti in modo opzionale, il depositario ${ }^{4}$, nonché la vigilanza dei fondi pensione volontari 5 .

Fin dalla entrata in vigore della legge legge n. 10197/2009 "In materia di fondi pensione volontari", il mercato delle pensioni complementari ha conosciuto una crescita positiva del numero dei soggetti autorizzati ad operare nel mercato. L'Autorità di Vigilanza Finanziaria ha approvato le autorizzazioni per tre società di amministrazione dei fondi pensione volontari e per le tre banche che fungono da depositario dei fondi pensione ${ }^{6}$. In questo mercato ci sono tre le società di amministrazione; Raiffeisen Invest, Sigal-Life Uniqa Group Austria, SiCred. Depositari del patrimonio dei fondi pensione per ogni società di amministrazione sono; First Investment Bank, Banka e Tiranes dhe Societé Générale Albania.

La nuova legge delle pensioni complementari, rappresenta un sistema nuovo della gestone, organizzazione ed funzionamento dei fondi pensione. Nel nuovo modello di organizzazione, si dedica particolare attenzione alla professionalità dei dirigenti delle società di amministrazione dei fondi pensione privati, tenendo conto la natura complessa di questi fondi $?$.

\section{La conformità alle direttive dell'Unione Europea, Banca Mondiale, OECD e IOPS.}

Anche se al momento dell'approvazione della nuova legge si è tenuto conto di tutte le Direttive dell'Unione Europea, non si riuscì ad emanare una legge in piena conformità alla Direttiva 2003/41/EC "In materia dell'attività e la vigilanza delle istituzioni che erogano le pensioni complementari" (IORP Directive). Questa è una delle Direttive più importanti sulle pensioni complementari, al quele tutti i paesi membri dell'Unione Europea devono adattare la legislazione in materia. Per questi motivi, il legislatore Albanese sta lavorando per il migloramento ed il ravvicinamento della legge in materia delle pensioni private ai prncipi di IORP.

L'Autorità di Vigilanza Finanziaria, applica anche la Direttiva 85/611/EEC del Consiglio Europeo in materia di coordinamento delle leggi, regole e disposizioni amministrative riguardo le iniziative per gli investimenti collettivi in titoli di credito trasferibili (UCITS); Inoltre, applica le Direttive di OECD ed a livelli soddisfacenti ha adempiuto gli obblighi derivanti dai principi IOPS sulle pensioni private complementari. Per questi motivi, la performance di questa Autorità è stata valutata positivamente dal Governo Albanese.

L'adesione dell'Albania all'Unione Europea è un processo dinamico che dipende dall'adempimento dei criteri di Copenaghen del 1993, e soprattutto dalla capacità del nostro paese ad adeguarsi al acquis communautaire e la sua piena trasposizione nella legislazione Albanese ${ }^{8}$.

\section{Conclusioni}

Il sistema pensionistico in generale e specialmente il sistema previdenziale complementare, rimangono una sfida per il nostro legislatore e le nostre Istituzioni. II Governo Albanese sta lavorando intensamente per la riforma di questo sistema, ma rimane sempre basso il livello di informazione dei cittadini sulle pensioni volontarie. A mio avviso, sarebbe necessaria una combinazione tra il sistema pensionistico obbligatorio con quello complementare o volontario, responsabilizzando in questo modo i cittadini per la risoluzione dei problemi sociali. Soltanto in questo modo ogni cittadino può assicurare una vita miglore dopo l'andata in pensione. L'approvazione e l'implementazione della riforma sarebbe una soluzione a lungo termine per il sistema pensionistico Albanese.

\footnotetext{
${ }^{4}$ II custode del patrimonio dei fondi pensione privati

5 /l fondo pensione è un conglomerato di capitali di proprietà dei membri di fondo pensione

${ }^{6}$ Annual Report of FSA (2012)

${ }^{7}$ Annual Report of FSA (2012)

${ }^{8}$ Annual Report of FSA (2012)
} 


\section{Bibliografia}

Dr. Kristaq Gjyli, Shkenca mbi financat dhe e drejta financiare, Tirane 2011.

Prof.Ass. Zhaklina Peto, E drejta e mbrojjes shoqerore, Tirane 2011.

Dan McGill, Kyle N. Brown, John J. Haley and Sylvester Schieber, Fundamentals of Private Pensions (February 2010).

John B. Shoven, Mark J. Warshawsky and William G. Gale, Private Pensions and Public Policies (May 2004).

Annual Report, FSA (2012)

Law 10 197, date 10.12.2009 "On voluntary pension funds".

DIRECTIVE 2003/41/EC OF THE EUROPEAN PARLIAMENT AND OF THE COUNCIL, of 3 June 2003, on the activities and supervision of institutions for occupational retirement provision.

COUNCIL DIRECTIVE 85/611/EEC, of 20 December 1985, on the coordination of laws, regulations and administrative provisions relating to undertakings for collective investment in transferable securities (UCITS).

Gjergji A. (2006),Does Albania Have a Developed Financial Market?! Non-banking sector, not functioning pillar of the system, Interdisciplinary Master on East European Studies \& Researches (MIREES) University of Bologna \& Istituto per l'Europa Centro Orientale, Bologna/ltaly, January 2006.

Xhumari M. (2010), Challenges facing the old-age pension system in Western Balkan, ISSA Conference "Emerging trends in times of instability: New challenges and opportunities for social security", 29 September - 1 October 2010, Luxembourg.

Andrews E. S., Rashid M. (1996), The Financing of Pension Systems in Central and Eastern Europe, World Bank Technical Paper No. 339.

www.amf.gov.al

www.issh.gov.al

www.ligjet.org 